Reprod. Nutr. Dévelop., 1982, 22 (2), 307-324.

\title{
Comportement alimentaire et utilisation digestive d'une ration de composition constante chez la vache laitière en fin de gestation et début de lactation
}

\author{
M. DOREAU, B. RÉMOND \\ avec la collaboration technique de Jeanne FLÉCHET, Renée LEFAIVRE, A. OLLIER \\ et Claire SORNET
}

Laboratoire de la Production laitière, I.N.R.A. Theix, 63110 Beaumont, France

Summary. - Feeding behaviour and digestion in dairy cows when fed a stable ration

Five cows, fitted with rumen and duodenal fistulas, were given ad libitum a small amount of hay and a stable ration of 60 p. 100 maize silage and 40 p. 100 concentrate from 4 weeks before calving to 6 weeks after. The pattern of feeding behaviour and of digestive processes was recorded over the whole period. The following results were obtained :

1) While the amounts of the ingested dry matter of the ration increased by $40 \mathrm{p} .100$, the digestibilities of dry matter, organic matter, crude fiber and starch decreased by an average of 7, 7, 10 and 5 points $p .100$ between 2 weeks before calving and the second week after. These values then increased by $3,3,3$ and 1 points $p .100$ between weeks 2 and 6 after calving (fig. 1).

2) The duration of intake and rumination increased rapidly while the rates varied little (fig. 2). At the same time, the number of meals, length of small meals and rumination periods increased, but the length of large meals was minimal around calving time (fig. 3). Consequently, the distribution of intake during the light/dark cycle was modified (fig. 4). 3) The preprandial ammonia content of the rumen liquor was minimal $(20 \mathrm{mg} / 1)$ the second week of lactation. The volatile fatty acid content and the proportions of different acids varied little throughout the period (fig. 5).

4) The flux of organic matter, non-ammonia nitrogen and starch to the duodenum increased rapidly but the amounts of organic matter digested in the rumen varied little, while those digested in the intestine multiplied almost 3 times, corresponding to a considerable decrease in rumen digestion (table 3, fig. 6). The amino acid composition of duodenal nitrogen varied little between the end of pregnancy and the onset of lactation (table 4).

\section{Introduction.}

Les semaines qui suivent la mise-bas de la vache laitière se caractérisent sur le plan nutritionnel par une augmentation brutale des besoins énergétiques, azotés et minéraux. L'accroissement des quantités ingérées, quoique rapide, est presque toujours insuffisant pour couvrir les besoins énergétiques avant un mois de 
lactation, même pour des rations de concentration énergétique élevée (Journet et Rémond, 1976). Cette médiocre capacité d'adaptation à l'évolution des besoins peut être liée à des particularités des ruminants, d'ordre comportemental (mastication mérycique) ou digestif (physiologie du rumen). On remarque en effet que l'accroissement des quantités ingérées après la mise-bas est plus lent chez les ruminants que chez les autres espèces, comme la ratte (Baumgardt, 1970) ou la lapine (Reyne et al., 1978).

L'effet de l'état physiologique sur le comportement alimentaire est mal connu. Campling (1966) et Suzuki, Fujita et Shinde (1969) notent une vitesse d'ingestion (quantité de matière sèche ingérée par unité de temps) plus élevée chez des vaches en gestation et surtout en lactation que chez des vaches vides et taries. Ils relient ce résultat à l'accroissement des quantités ingérées ; une telle liaison est en effet établie sur moutons à l'entretien (Dulphy, 1971). Les résultats de Journet et Rémond (1976) sur régime riche en foin permettent de plus de mettre en évidence un effet propre du stade physiologique : entre le premier et le troisième mois de lactation, les durées et vitesses d'ingestion varient alors que les quantités ingérées n'augmentent que peu.

La digestibilité d'une même ration est plus faible en fin de gestation qu'en période de tarissement chez la vache (de 2 à 5 points p. 100 environ : Lamberth, 1969 ; Flatt, Moe et Moore, 1969 ; Everson et al., 1976). Ceci semble être dû à une accélération du transit provoquée par la diminution du volume du rumen, comprimé par le fœetus, les annexes et éventuellement les réserves adipeuses : cet effet apparaît clairement chez la brebis (Weston, 1979). Une digestibilité encore plus faible en début de lactation (jusqu'à 10 points p. 100) par rapport à l'entretien a fréquemment été observée pour différentes rations (Flatt, Coppock et Moore, 1965 ; Wheeler, Noller et Coppock, 1975). Elle a essentiellement été reliée à l'accroissement des quantités ingérées : l'influence du niveau d'ingestion sur la digestibilité d'une ration est bien établie (Tyrrell et Moe, 1975). La cause principale serait également l'augmentation de la vitesse de passage des digesta, liée positivement aux quantités ingérées (Campling, Freer et Balch, 1961), se traduisant notamment par une diminution de la part du rumen dans la digestion (Ulyatt, Blaxter et Macdonald, 1967).

Les éventuelles modifications comportementales et digestives intervenant en début de lactation peuvent, en agissant sur les quantités ingérées et la digestibilité, modifier l'importance et la durée du déficit énergétique à cette période. II importe donc d'en connaître la cinétique avec précision. Aussi, nous avons étudié les variations de l'utilisation d'une même ration par la vache laitière pendant le dernier mois de gestation et les six premières semaines de lactation : activités alimentaires, digestibilité, digestion au niveau du rumen. Cette ration se caractérisait par son aptitude à être ingérée en quantités élevées et une teneur en azote fermentescible permettant théoriquement la satisfaction des besoins en azote des microorganismes du rumen (cf. Jarrige et al., 1978). Sa composition très peu variable facilitait l'interprétation des résultats en supprimant les effets d'une modification du pourcentage d'aliment concentré. 


\section{Matériel et méthodes.}

\section{- Animaux et régime.}

L'essai a été conduit sur 5 vaches issues de croisement Holstein $\times$ FFPN et de numéro de lactation compris entre 2 et 5 , pourvues d'une large canule du rumen et d'une canule simple du duodénum proximal. Leur poids moyen après vêlage était de $624 \mathrm{~kg}$. Il a été réalisé sur 2 années consécutives $(2$ vaches puis 3 vaches).

L'expérience s'est déroulée de 4 semaines avant à 6 semaines après vêlage. Durant cette période, les animaux ont reçu le même régime comprenant du foin de prairie naturelle de $2^{\mathrm{e}}$ coupe $(1,7 \mathrm{~kg}$ de matière sèche $/ \mathrm{j})$, un mélange d'ensilage de maïs et d'aliment concentré (respectivement 60 et 40 p. 100 sur la base de la matière sèche), d'urée et d'un complément minéral $(1,2$ p. 100 chacun du mélange précédent), offert ad libitum. La nature et la composition chimique de ces aliments sont précisées au tableau 1.

\section{TABLEAU 1}

Composition chimique et valeur nutritive des aliments distribués (en p. 100 de la matière sèche)

\begin{tabular}{|c|c|c|c|c|c|c|c|}
\hline & \multicolumn{4}{|c|}{ Teneur en p. 100 de la MS } & \multicolumn{3}{|c|}{ Valeur nutritive } \\
\hline & $\begin{array}{c}\text { Matière } \\
\text { organique }\end{array}$ & $\begin{array}{c}\text { Cellulose } \\
\text { brute }\end{array}$ & Amidon & $\begin{array}{c}\text { Matières } \\
\text { azotées }\end{array}$ & UFL & $\begin{array}{c}\text { PDIN } \\
(\mathrm{g})\end{array}$ & $\begin{array}{l}\text { PDIE } \\
(\mathrm{g})\end{array}$ \\
\hline $\begin{array}{l}\text { Foin } \ldots \ldots \ldots \\
\text { Ensilage de maïs } \ldots \ldots \\
\text { Aliment concentré (i) } \ldots\end{array}$ & $\begin{array}{l}90,3 \\
95,3 \\
93,2\end{array}$ & $\begin{array}{r}27,0 \\
18,3 \\
9,1\end{array}$ & $\begin{array}{l}- \\
32,1 \\
36,8\end{array}$ & $\begin{array}{r}15,0 \\
7,5 \\
18,3\end{array}$ & $\begin{array}{l}0,79 \\
0,85 \\
1,06\end{array}$ & $\begin{array}{r}103 \\
47 \\
137\end{array}$ & $\begin{array}{r}96 \\
69 \\
157\end{array}$ \\
\hline
\end{tabular}

(1) Composition : maïs (40 p. 100), pulpes de betteraves (16 p. 100), orge (14 p. 100), tourteau de soja tanné (10 p. 100), tourteau de colza tanné (10 p. 100), luzerne déshydratée (8 p. 100), minéraux (2 p. 100).

Le foin était offert à $9 \mathrm{~h}$. A partir de $9 \mathrm{~h} 30$, les refus étaient enlevés et pesés. Le reste de la ration (ensilage de maïs, aliment concentré, urée, complément minéral) était mélangé et distribué en deux fractions égales aux environs de $9 \mathrm{~h} 30$ et à $17 \mathrm{~h}$. Les quantités distribuées étaient calculées de manière à obtenir une proportion de refus voisine de 15 p. 100 sur l'ensemble de la journée.

\section{- Mesures.}

Quantités ingérées et production. - Les quantités ingérées ont été mesurées tous les jours et la teneur en matière sèche déterminée chaque jour à l'étuve ventilée à $80^{\circ} \mathrm{C}$. La correction de la teneur en matière sèche des ensilages pour la perte de composés volatils a été effectuée selon Dulphy, Demarquilly et Henry (1975). La production laitière a été mesurée tous les jours et les teneurs du lait en 
protéines et en matières grasses avec un appareil "Milko tester " tous les jours lors de la première semaine de lactation et 2 fois par semaine ultérieurement sur un échantillon pondéré des 2 traites quotidiennes.

Comportement alimentaire. - Les activités alimentaires ont été enregistrées 5 jours par semaine par la méthode de Ruckebusch (1963) : les mouvements de la mâchoire étaient transmis par un ballonnet rempli de mousse de polyuréthane et maintenu en position sous-maxillaire à un capteur pneumatique (tambour de Marey) relié à un enregistreur graphique.

Par convention, on a appelé repas toute période d'ingestion de plus de 10 min., éventuellement entrecoupée d'arrêts de moins de $10 \mathrm{~min}$. Les deux repas suivant les distributions d'aliments ont été appelés grands repas, et les autres petits repas. La nature des aliments consommés (foin ou mélange ensilage de maïs-aliment concentré) n'a pas été distinguée.

Digestibilité. - Les animaux étaient installés en permanence dans des stalles de digestibilité sur tapis de caoutchouc, sauf pendant 3 jours au maximum, lors de la parturition.

La digestibilité de la ration a été mesurée chaque semaine pendant l'ensemble de l'essai, par collecte des fèces durant 5 jours. Nous avons observé un décalage de $24 \mathrm{~h}$ entre la mesure des quantités ingérées et celle des quantités excrétées. La composition de la ration ingérée était calculée pour chaque vache d'après celles des aliments distribués et refusés. L'échantillonnage des fèces pour analyses, effectué chaque jour, était proportionnel aux quantités excrétées. L'azote était dosé sur la matière fraîche par la méthode de Kjeldahl, la cellulose brute par la méthode de Weende, les cendres par minéralisation à $550^{\circ} \mathrm{C}$ pendant $12 \mathrm{~h}$ et l'amidon par la méthode de Thivend, Mercier et Guilbot (1972) à la glucoamylase.

Fermentations dans le rumen. - Des prélèvements de la phase liquide du contenu ruminal ont été effectués dans le sac ventral deux jours par semaine à $8 \mathrm{~h}$ $30,11 \mathrm{~h}, 13 \mathrm{~h}, 14 \mathrm{~h}, 16 \mathrm{~h} 30$. Après mesure du $\mathrm{pH}$, un échantillon moyen de la journée a été réalisé pour détermination des acides gras volatils par chromatographie en phase gazeuse. La teneur en ammoniaque a été déterminée par une méthode utilisant la réaction de Berthelot (Van Eenaeme et al., 1969), sur les prélèvements de $8 \mathrm{~h} 30$ et $11 \mathrm{~h}$ (sur 3 vaches).

Passage des digesta au niveau du duodénum. - Le flux de nutriments a été estimé sur 3 animaux par l'intermédiaire d'un marqueur, l'oxyde de chrome, introduit par la canule du rumen à chacune des 2 distributions journalières d'aliments sous forme de granulés ( 25 p. 100 d'oxyde de chrome, adsorbé sur poudre de cellulose : Tisserand, Coleou et Zelter, 1962) à raison de $70 \mathrm{~g}$ par distribution.

Cinq prélèvements journaliers $(8 \mathrm{~h} 30,11 \mathrm{~h}, 13 \mathrm{~h}, 14 \mathrm{~h} \mathrm{30}, 16 \mathrm{~h} 30$ ) ont été réalisés pour chaque semaine pendant 3 jours consécutifs. Sur l'échantillon moyen on a dosé les cendres, l'azote total et ammoniacal et l'amidon par les méthodes précitées. L'oxyde de chrome a été oxydé par un mélange nitroperchlorique, puis dosé colorimétriquement d'après une méthode dérivée de Mathieson (1970). Les flux de matière sèche et de nutriments passant par le duodénum ont été calculés en estimant que la quantité de marqueur passant par jour dans le duodénum était égale à celle récupérée dans les fèces. Sur les échantillons cor- 
respondant à 2 semaines avant et 2 semaines après le vêlage, on a aussi déterminé la composition en acides aminés par une méthode dérivée de Moore, Spackmann et Stein (1958) après extraction des matières azotées selon Pion, Champredon et Thivend (1973).

\section{Résultats.}

Quantités ingérées, production laitière et digestibilité (fig. 1):

Les quantités de matière sèche ingérées, élevées en fin de gestation lenviron $12 \mathrm{~kg}$ ) diminuent de $22 \mathrm{p}$. 100 environ durant les deux semaines précédant la mise bas. L'augmentation est très rapide pendant les 10 premiers jours de lactation (de $5 \mathrm{~kg}$ de MS environ, soit $72 \mathrm{p}$. 100 de l'accroissement total) puis se stabilise à partir de la $4^{\mathrm{e}}$ semaine aux alentours de $17 \mathrm{~kg}$. L'une des vaches présente une dimi-

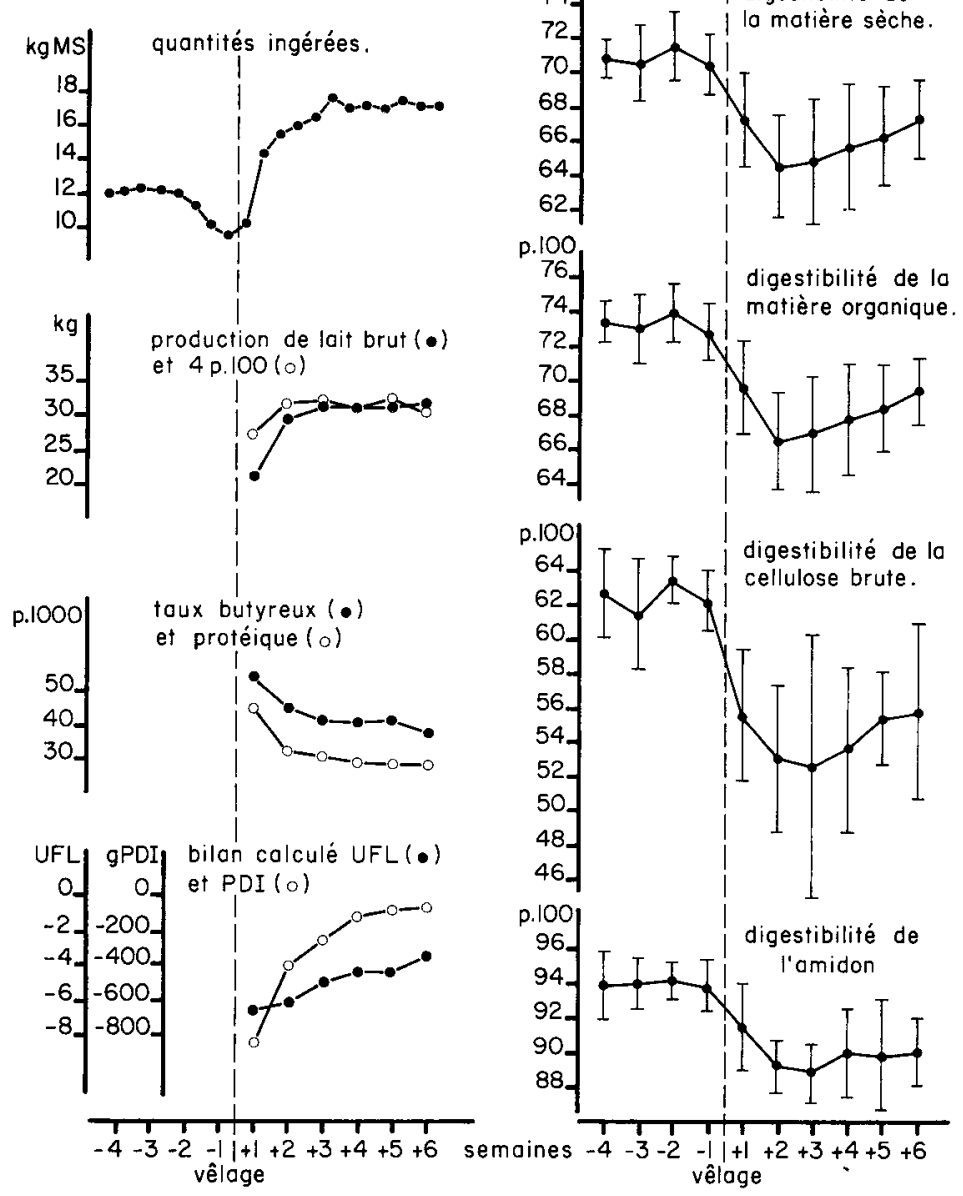

FIG. 1. - Quantités ingérées, production laitière et digestibilité de différents constituants de la ration. 
nution brutale et temporaire (4 jours) de l'ingestion en $4^{e}$ semaine, sans effet rémanent apparent pour la suite de l'expérience. Les jours correspondant à cette période ont été exclus de la moyenne hebdomadaire des quantités ingérées de cette vache et la période de digestibilité supprimée. La consommation de foin est voisine de 1,3 kg de MS par jour, quel que soit le stade physiologique ; la proportion de foin dans la ration est donc passée de 11 p. 100 environ en fin de gestation à 8 p. 100 environ en début de lactation.

La production laitière brute est de $31 \mathrm{~kg} / \mathrm{j}$ en moyenne dès la $3^{\mathrm{e}}$ semaine de lactation. Elle diminue légèrement en $4^{e}$ semaine en raison d'une chute de production de $9 \mathrm{~kg}$ de lait brut de la vache ayant manifesté une chute d'ingestion; sa production laitière $n^{\prime}$ a plus augmenté après le retour à un niveau d'ingestion normal.

Les digestibilités de la matière sèche, de la matière organique, de la cellulose brute et de l'amidon diminuent fortement en un laps de temps réduit, entre la $2^{\mathrm{e}}$ semaine avant et la $2^{\mathrm{e}}$ semaine après le vêlage. Entre les $3^{\mathrm{e}}$ et $6^{\mathrm{e}}$ semaines de lactation, les digestibilités augmentent modérément. Ces variations sont globalement significatives à un seuil de 1 p. 100 (analyse de variance sur les valeurs hebdomadaires). Les différences entre vaches sont plus importantes en début de lactation qu'en fin de gestation, en particulier pour la digestibilité de la cellulose brute, dont l'écart-type est multiplié par 3.

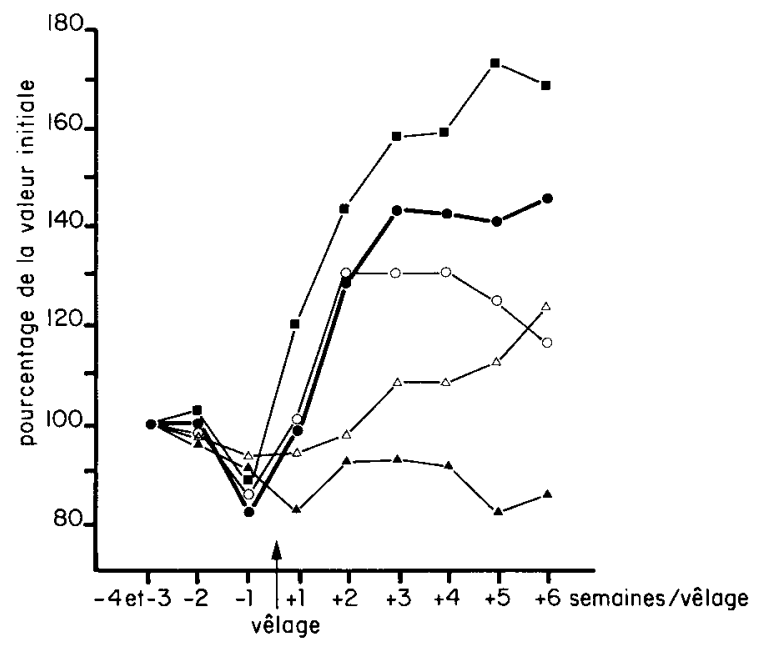

FIG. 2. - Evolution comparée des quantités ingérées et des durées et vitesses d'ingestion et de rumination.

- : Quantités ingérées (base $100=11,8 \mathrm{~kg} \mathrm{MS}$ ) ;

Durée d'ingestion (base $100=209 \mathrm{~min}$.); Durée de rumination (base $100=379 \mathrm{~min}$.) ; $\mathbf{\Delta}$ : Vitesse d'ingestion (base $100=53,8 \mathrm{~g}$ $\mathrm{MS} / \mathrm{min}$ ) : $\triangle:$ Vitesse de rumination (base $100=31,0 \mathrm{~kg} \mathrm{MS} / \mathrm{min}$.).

Comportement alimentaire (fig. 2 et 3 ; tabl. 2) :

L'accroissement des quantités ingérées en début de lactation se traduit au niveau des activités alimentaires par une augmentation rapide de la durée de mas- 
tication (ingestion + rumination) : de moins de $600 \mathrm{~min} . / \mathrm{j}$ en fin de gestation à $800 \mathrm{~min} . / \mathrm{j}$ dès la $3^{e}$ semaine de lactation. La durée d'ingestion augmente régulièrement au moins jusqu'à la $5^{e}$ semaine de lactation, alors que la durée de rumination n'augmente que pendant les deux premières semaines. La vitesse de mastication (quantité de matière sèche mastiquée par unité de temps) est peu modifiée entre la fin de la gestation et les six premières semaines de lactation : les vitesses d'ingestion et de rumination sont minimales à proximité immédiate du vêlage, seule la vitesse de rumination augmente ensuite. Leurs variations sont cependant beaucoup plus faibles que celles des durées d'ingestion et de rumination.

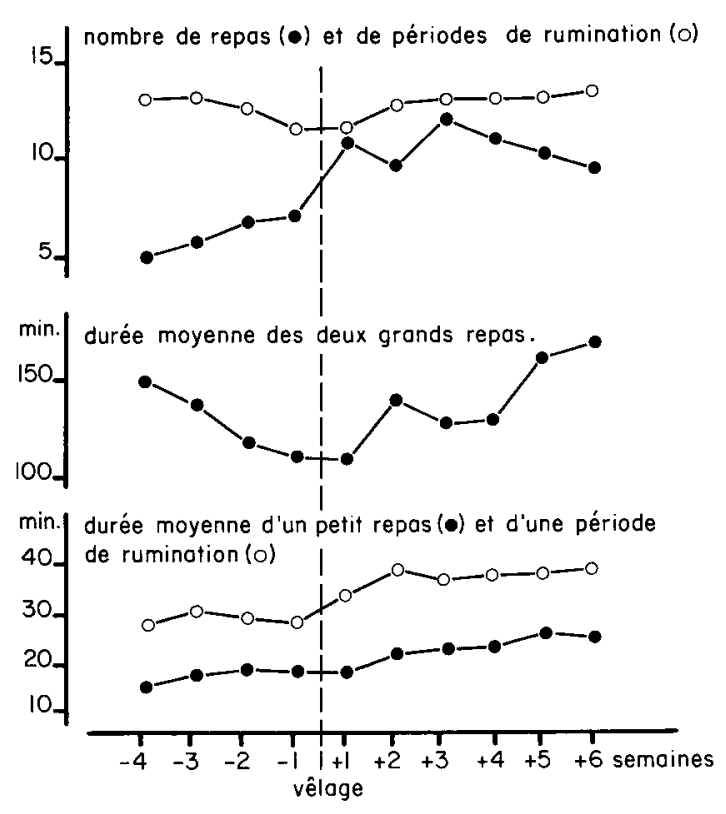

FIG. 3. - Fractionnement des activités alimentaires.

L'accroissement de la durée d'ingestion s'effectue au moyen d'une augmentation significative du nombre de repas et de la durée des petits repas. L'augmentation du nombre de repas commence avant le vêlage, alors que les quantités ingérées diminuent. En revanche, la durée des grands repas diminue durant les deux dernières semaines de gestation, est minimale au moment du vêlage, puis augmente à partir de la $2^{\mathrm{e}}$ semaine de lactation. Ceci conduit à une modification de la répartition de l'ingestion au cours du nycthémère (fig. 4) : alors qu'en fin de gestation l'ingestion est concentrée sur les grands repas, en début de lactation elle se déroule en partie au cours de la journée ou au début de la nuit, en petits repas. L'augmentation de la durée de rumination est due à l'accroissement de la 
durée de chaque période de rumination, mais non à celui du nombre journalier de périodes, qui diminue même d'une unité environ à proximité immédiate du vêlage.

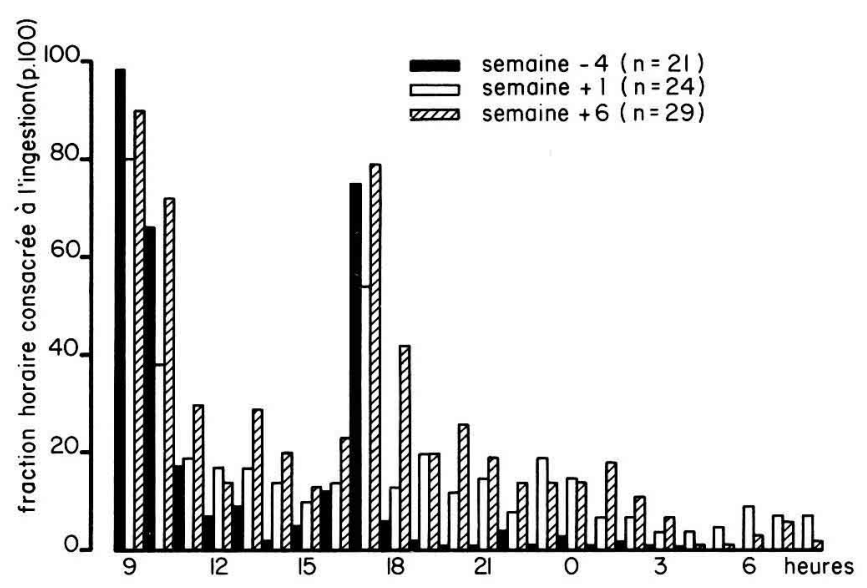

Fig. 4. - Répartition de l'ingestion au cours du nycthémère. Influence du stade physiologique.

Lorsque l'on considère les résultats journaliers, il apparaît que seules les durées d'activités alimentaires (ingestion, rumination et mastication) sont étroitement corrélées aux quantités ingérées. Une comparaison entre les parts de variation dues au stade physiologique (intégrant l'augmentation des quantités ingérées) et à l'animal, met en évidence un effet important du stade physiologique sur les durées d'activité et le nombre de repas (tabl. 2).

\section{Digestion}

Digestion dans le rumen (fig. 5). - Le pH du liquide du rumen, mesuré avant la distribution d'aliments, varie peu au cours de l'essai, mis à part dans la semaine suivant le vêlage. En revanche, les $\mathrm{pH}$ mesurés 2 et $4 \mathrm{~h}$ après la distribution d'aliments diminuent en moyenne de 0,2 point entre la fin de gestation et la $4^{\mathrm{e}}$ semaine de lactation. Les deux semaines entourant le vêlage se caractérisent par un minimum de $\mathrm{pH} 2 \mathrm{~h}$ après la distribution d'aliments et non $4 \mathrm{~h}$ après.

La teneur en acides gras volatils totaux, comprise entre 70 et $80 \mathrm{mM} / \mathrm{I}$, :est minimale aux alentours du vêlage ; les variations en fonction du stade physiologique ne sont cependant pas significatives. La proportion des différents acides gras volatils est peu variable ; il apparaît cependant une tendance non significative (2 points p. 100 environ) à la diminution de l'acétate au profit du propionate dans les premières semaines de lactation.

La teneur en ammoniaque avant la distribution d'aliments est maximale en dernière semaine de gestation $(80 \mathrm{mg} / \mathrm{l})$ puis diminue très rapidement jusqu'à 20 


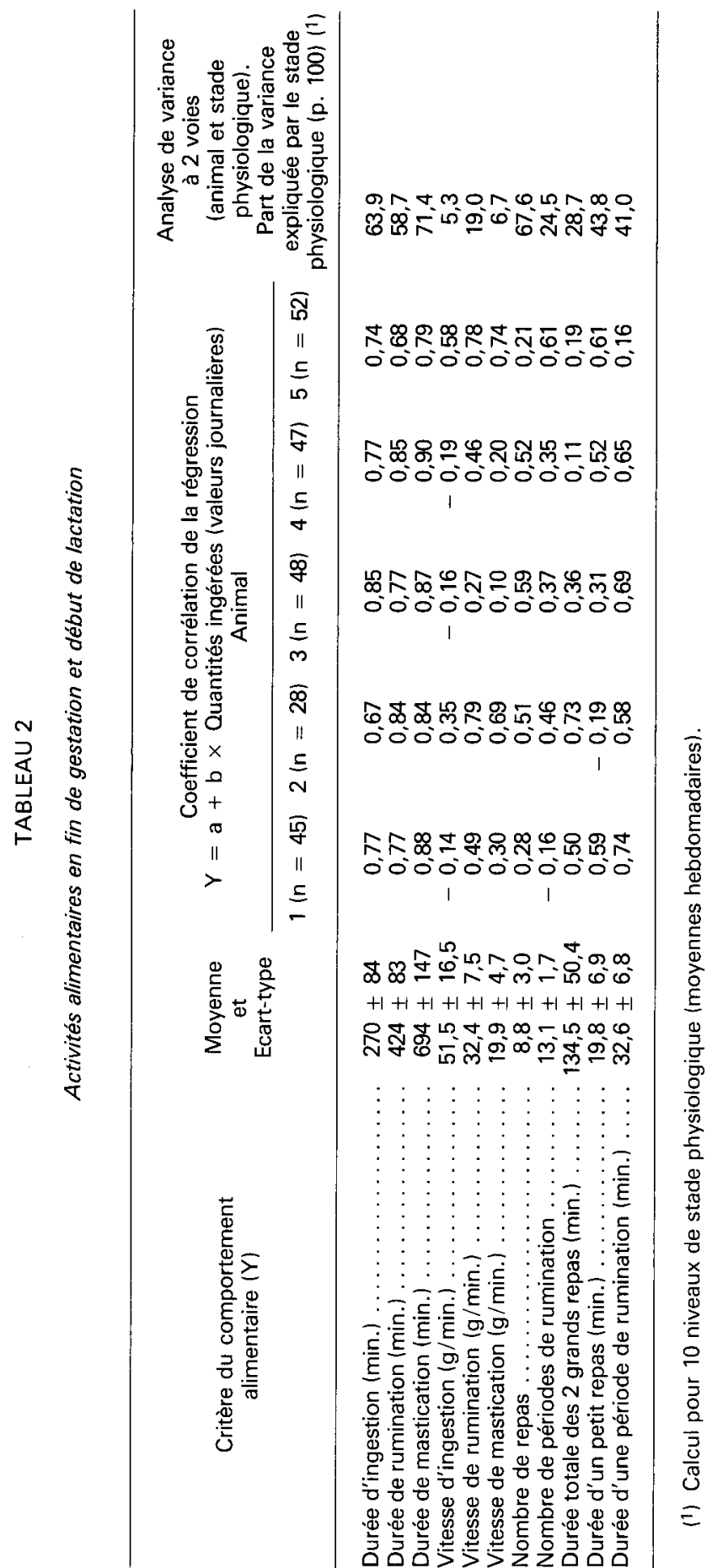




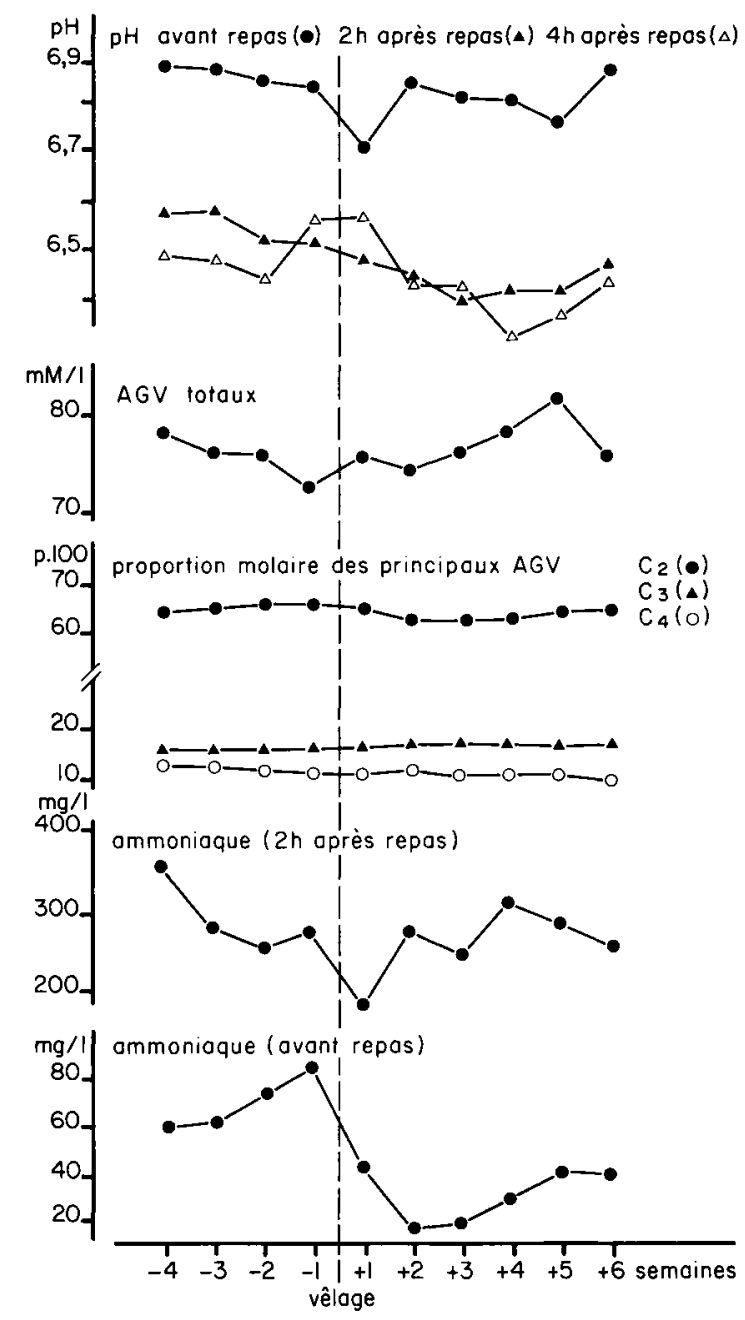

FIG. 5. $-p H$ et produits terminaux du liquide du rumen.

$\mathrm{mg} / \mathrm{l}$ en $2^{\mathrm{e}}$ semaine de lactation et augmente modérément à partir de la $4^{\mathrm{e}}$ semaine ; en revanche, prise $2 \mathrm{~h}$ après la distribution d'aliments, elle est élevée et n'évolue pas significativement.

Bilan de la digestion au niveau du duodénum (tabl. 3 ; fig. 6 et 7). - Les récupérations d'oxyde de chrome dans les fèces ont été en moyenne de 90 p. 100. Leur variabilité n'a pas dépendu du stade physiologique. Par ailleurs, au cours d'une même semaine, les variations d'un jour à l'autre de la concentration en oxyde de chrome dans le contenu du duodénum ont été élevées, principalement dans les trois premières semaines de lactation, probablement en raison d'un nom- 
TABLEAU 3

Digestion de la matière organique, de l'azote et de l'amidon mesurée par l'intermédiaire des flux duodénaux

Semaines par rapport au vêlage

Nombre de données (vache $\times$ Période) $\ldots . .$. . Matière organique :

- Quantités ingérées $(\mathrm{kg}) \ldots \ldots \ldots \ldots \ldots$

- Flux duodénal $(\mathrm{kg}) \ldots \ldots \ldots \ldots \ldots \ldots \ldots$

- Quantités excrétées $(\mathrm{kg}) \ldots \ldots \ldots \ldots \ldots$. . . .

- Digestibilité totale (p. 100) ...........

- Digestibilité dans le rumen (p. 100 de Azote :

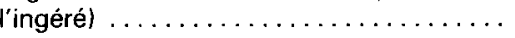

- Azote ingéré $(g) \ldots \ldots \ldots \ldots \ldots \ldots$

- Flux duodénal d'azote non ammoniacal (g) $\ldots \ldots \ldots \ldots \ldots \ldots \ldots \ldots \ldots$

- Azote non ammoniacal duodénal/azote ingéré $\ldots \ldots \ldots \ldots \ldots \ldots \ldots \ldots$ Amidon :

- Quantités ingérées (g)

4047

- Flux duodénal (g) ................. 563

- Quantités excrétées $(\mathrm{g}) \ldots \ldots \ldots \ldots \ldots . . . .242$

- Digestibilité totale $(p .100) \ldots . . \ldots \ldots$. . .

- Digestibilité dans le rumen (p. 100 de l'ingéré)

5

12,1

4,6

3,1

74,4

61,7

294

251

86,1
Les moyennes de chaque colonne sont effectuées d'abord intra-vache, puis entre vaches.

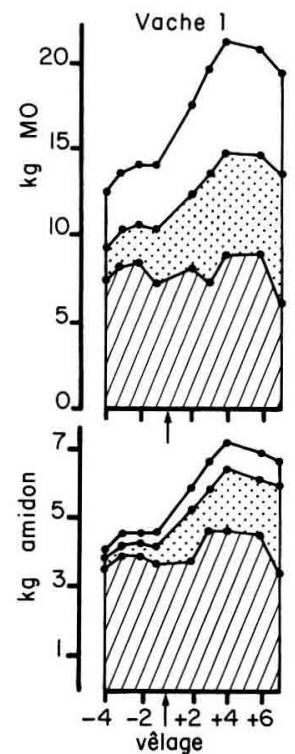

Vache 2

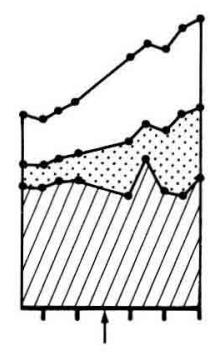

FIG. 6. - Quantités de matière organique et d'amidon ingérées et digérées dans le rumen et l'intestin.

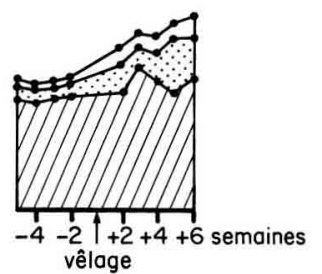

quantités non digérées.

quantités digérées dans l'intestin.

quantités digérées dons le rumen.

$\begin{array}{cccc}4205 & 5290 & 5839 & 6192 \\ 649 & 1686 & 1690 & 2342 \\ 240 & 527 & 598 & 652 \\ 94,3 & 90,0 & 89,8 & 89,5\end{array}$

$84,6 \quad 68,1 \quad 71,1 \quad 62,2$

\section{4}

8,4

$\begin{array}{llll}5,2 & 8,8 & 9,9 & 11,1\end{array}$

$\begin{array}{llll}3,2 & 4,5 & 5,5 & 5,6\end{array}$

$\begin{array}{llll}75,2 & 68,5 & 69,6 & 69,7\end{array}$

$60,0 \quad 45,3 \quad 45,0 \quad 40,2$

$\begin{array}{llll}320 & 424 & 452 & 480 \\ 283 & 374 & 476 & 499\end{array}$




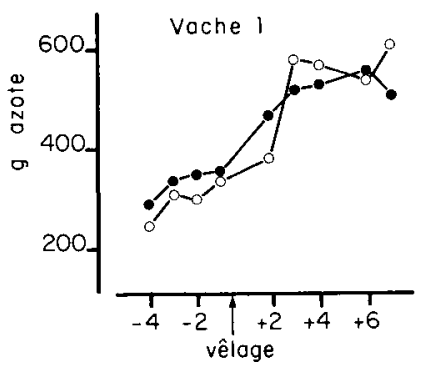

vache 2

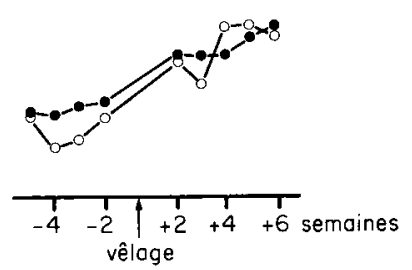

FIG. 7. - Flux d'azote non ammoniacal arrivant au duodénum et quantités d'azote ingéré.

- : Azote ingéré ; $O$ : Azote non ammoniacal arrivant au duodénum.

bre insuffisant de prélèvements et d'une irrégularité accrue du transit digestif juste après le vêlage. Les flux de matière sèche calculés au cours de plusieurs semaines successives du début de la lactation ont présenté une forte variabilité, surtout pour un animal que nous avons dû éliminer. Les résultats ne portent donc que sur 2 animaux.

L'augmentation des quantités de matière organique ingérées se traduit par l'accroissement important du flux duodénal, multiplié par 2. Alors que les quantités digérées dans le rumen évoluent peu, les quantités digérées dans l'intestin augmentent rapidement et sont multipliées par 3 . La part de la digestion dans le rumen, très élevée en fin de gestation (environ 80 p. 100), diminue rapidement en début de lactation, devenant proche de 60 p. 100 dans le $2^{\mathrm{e}}$ mois de lactation.

\section{TABLEAU 4}

Composition en acides arninés des contenus de duodénum avant et après vélage

\begin{tabular}{|c|c|c|}
\hline $\begin{array}{l}\text { Teneur en acides aminés } \\
\quad(\mathrm{en} \mathrm{g} / 16 \mathrm{~g} \mathrm{~N})\end{array}$ & $\begin{array}{l}2 \text { semaines } \\
\text { avant vêlage }\end{array}$ & $\begin{array}{l}2 \text { semaines } \\
\text { après vêlage }\end{array}$ \\
\hline 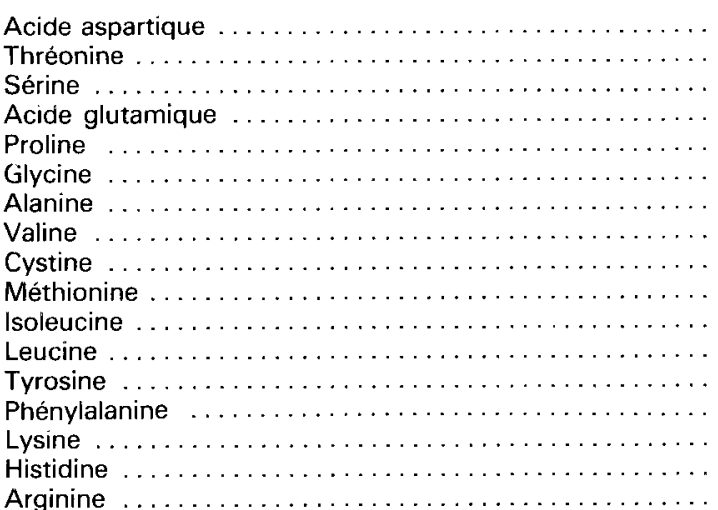 & $\begin{array}{r}9,45 \\
4,56 \\
4,48 \\
13,52 \\
4,41 \\
4,95 \\
5,55 \\
4,73 \\
1,93 \\
1,44 \\
4,22 \\
7,25 \\
4,18 \\
4,51 \\
5,86 \\
2,15 \\
2,27\end{array}$ & $\begin{array}{r}9,67 \\
4,35 \\
4,39 \\
13,76 \\
4,56 \\
5,44 \\
5,43 \\
4,85 \\
1,83 \\
1,48 \\
4,31 \\
7,25 \\
4,36 \\
4,74 \\
5,84 \\
2,09 \\
4,49\end{array}$ \\
\hline
\end{tabular}


L'augmentation importante du flux duodénal d'azote non ammoniacal entre la fin de gestation et le début de lactation est supérieure à celle de l'azote ingéré ; le rapport azote non ammoniacal passant par le duodénum/azote ingéré, voisin de 0,9 en fin de gestation, devient en moyenne supérieur à 1 à partir de la $3^{\mathrm{e}}$ semaine de lactation.

La composition en acides aminés ne change pas entre 15 jours avant et 15 jours après la mise bas, mise à part une légère augmentation de la glycine (tabl. 4), alors que les compositions en acides aminés théorique des microorganismes et estimée des ingesta sont très différentes : à titre d'exemple : les teneurs de la ration, des protozoaires et des bactéries en g pour $16 \mathrm{~g}$ d'azote sont évaluées respectivement à 3,$4 ; 1,8$ et 1,4 pour l'histidine.

\section{Discussion.}

\section{Comportement alimentaire.}

Les modifications des activités alimentaires aux alentours du vêlage peuvent être reliées à deux facteurs principaux : 1) l'accroissement rapide des quantités ingérées en début de lactation. Dans cet essai, il se traduit par l'augmentation de la durée d'ingestion et de rumination, grâce à une augmentation du nombre et de la durée des petits repas et de la durée des périodes de rumination. En revanche, le nombre de périodes de rumination est particulièrement constant, sauf à proximité immédiate du vêlage : Freer, Campling et Balch (1962) avaient de même observé chez la vache que le nombre de périodes de rumination était une caractéristique individuelle et dépendait peu des quantités ingérées; 2 ) les variations du volume du rumen. Les travaux de Fell et al. (1972) puis Hartnell et Satter (1978) laissent penser qu'il diminue lentement en fin de gestation, puis augmente brutalement après la mise bas (effet de la compression exercée par le fœtus et les annexes embryonnaires, puis de la dépression causée par leur expulsion), et augmente certainement modérément ensuite (effet possible du développement tissulaire du rumen et de la mobilisation des réserves adipeuses abdominales). Or, nous observons des variations correspondantes de la durée des grands repas. Le volume du rumen agirait ainsi sur le rassasiement à court terme.

La comparaison entre nos résultats et ceux de Journet et Remond (1976) sur un régime riche en foin et un régime broyé aggloméré montre que l'évolution des activités alimentaires aux alentours du vêlage ne suit pas de règle générale : ainsi, la durée de rumination n'augmente pas avec le régime riche en foin, probablement en raison d'une durée déjà très élevée avant vêlage ; avec le régime broyé aggloméré, les premiers jours de lactation se caractérisent par une augmentation très rapide de la durée de rumination et une baisse de la vitesse de rumination ; les résultats disponibles, trop peu nombreux, ne permettent pas d'établir un schéma global d'évolution des durées et vitesses d'ingestion et de rumination.

\section{Digestion.}

Le problème méthodologique majeur de cet essai est constitué par l'estimation des flux duodénaux. La limitation des prélèvements de contenus de duodé- 
num à la phase diurne a permis de ne pas perturber le comportement alimentaire des animaux. En revanche, elle a pu introduire une erreur qui, si elle a été reconnue importante chez le mouton (Laughren et Young, 1979), semble plus limitée chez la vache (Van't Klooster et al., 1972 ; Vérité, communication personnelle). Si la technique de prélèvement laisse cependant subsister une marge d'incertitude sur les valeurs absolues des flux, il est probable que son influence sur les évolutions en fonction du stade physiologique est moindre.

La chute importante de digestibilité de la matière organique est explicable en grande partie par l'augmentation des quantités ingérées. Cependant, l'augmentation du niveau d'alimentation d'une unité (ce qui correspond dans notre essai à l'évolution entre la fin de gestation et la $3^{e}$ semaine de lactationl, n'a pas entraîné une diminution de digestibilité supérieure à 5 points pour des régimes à base d'ensilage de maïs comprenant entre 30 et 60 p. 100 d'aliment concentré dans les essais de Tyrrell et Moe (1972) et Dulphy et Carle (résultats non publiés). II est probable que d'autres facteurs ont accentué la chute de digestibilité. Ainsi, l'adaptation de la physiologie ruminale, par exemple du développement épithélial du rumen mis en évidence chez la brebis par Fell et al. (1972), a pu être plus lente que l'augmentation des quantités ingérées. Le déficit azoté de l'animal dans les premières semaines de lactation peut également contribuer à cette chute (Rémond, résultats non publiés). Ces facteurs expliqueraient en outre dans cet essai la légère augmentation de digestibilité à partir de la $3^{e}$ semaine de lactation.

En fin de gestation, la proportion de matière organique digérée dans le rumen a été élevée ; seuls Siebert et Allden (1979) signalent des valeurs aussi élevées, sur un régime à base d'herbe pâturée. En début de lactation, les valeurs que nous obtenons sont plus comparables aux résultats habituellement observés. La diminution de la proportion digérée dans le rumen peut s'expliquer en partie par une diminution du temps de séjour des digesta dans ce compartiment consécutive à l'augmentation du niveau d'ingestion. Elle est probablement accentuée par un niveau azoté insuffisant, qui se traduit par une teneur en ammoniaque du rumen très faible. En fin de gestation, la teneur pré-prandiale, donc proche du minimum, en ammoniaque du rumen, est supérieur à $60 \mathrm{mg} / \mathrm{l}$; cette concentration dénote un apport élevé d'azote par rapport à l'énergie disponible pour les microorganismes et ne devrait pas ou peu limiter la synthèse de protéines microbiennes (Satter et Slyter, 1974 ; Kang-Meznarich et Broderick, 1980). En début de lactation, la teneur en ammoniaque du rumen diminue fortement ainsi que l'urémie, dont l'évolution est décrite par ailleurs (Doreau, en préparation). Or, la ration que nous avons utilisée apporte en $2^{\mathrm{e}}$ semaine de lactation environ $135 \mathrm{~g}$ de matières azotées fermentescibles par $\mathrm{kg}$ de matière organique digestible, ce qui aurait dû assurer la couverture des besoins azotés des microorganismes. Ceci permet de penser que les besoins en matières azotées fermentescibles sont accrus dans les cas de déficit azoté, probablement en raison d'un transfert de l'urée sanguine au tractus digestif quantitativement moins important (Cocimano et Leng, 1967). Cependant, l'augmentation du rapport entre l'azote non ammoniacal arrivant au duodénum et l'azote ingéré entre la fin de gestation et le début de lactation semble indiquer une utilisation effective de l'azote provenant du compartiment sanguin. Les équations de prédiction du flux d'azote non ammoniacal parvenant au 
duodénum, établies par Journet et Vérité (1979), ont été appliquées à notre essai. Ces équations tiennent compte de la matière organique digestible et de la quantité d'azote non dégradable ingérées. Les flux que nous avons obtenus sont en moyenne 15 p. 100 inférieurs en fin de gestation et 10 p. 100 supérieurs en début de lactation aux flux prédits. Ces écarts, peu importants compte tenu du seuil de précision de nos mesures, peuvent être dus à l'effet du recyclage de l'azote alors que Journet et Vérité ont effectué leurs mesures en cours de lactation.

Les proportions respectives d'azote d'origine alimentaire et microbienne varient peu. Or, il a été montré que la part d'azote d'origine alimentaire augmentent en même temps que le niveau d'ingestion (Tamminga, van der Koelen et van Vuuren, 1979) et lorsque le temps de séjour des particules solides diminue (Crawford, Hoover et Junkins, 1980). Nos résultats pourraient s'expliquer par une efficacité accrue de la synthèse microbienne, liée à un renouvellement plus rapide du liquide du rumen (Isaacson et al., 1975) et prévue par le modèle de Baldwin, Koong et Ulyatt (1977), ou par la proportion importante dans la ration de l'azote en provenance des tourteaux tannés non dégradables dans le rumen. Ces deux facteurs contribuent d'ailleurs à diminuer la teneur en ammoniaque du rumen. Dans le cas présent, la synthèse microbienne pourrait être à la fois accrue grâce à une meilleure utilisation de l'azote et limitée par la teneur en azote de la ration.

La concentration des AGV du rumen ne varie pas au cours de notre essai ; on peut cependant penser que l'augmentation probable du volume du rumen en début de lactation implique un léger accroissement de la quantité d'AGV présente. Cette relative stabilité est confirmée par celle de la quantité de matière organique digérée dans le rumen. La faible variation de la proportion molaire des différents $A G V$ indique que la dégradation des différentes sources glucidiques est peu modifiée. Ces résultats peuvent être comparés à ceux obtenus jusqu'à présent pour des régimes de composition fixée, et qui semblent contradictoires : l'augmentation du niveau alimentaire provoquerait une faible diminution relative de l'acétate au profit du propionate (Sutton et al., 1977) ou du butyrate (Bath et Rook, 1963) ; l'interprétation de ces résultats est délicate car la composition du mélange des AGV dépend des vitesses de disparition des particules solides et de renouvellement du liquide du rumen (Harrison et al., 1975 ; Crawford, Hoover et Knowlton, 1980).

L'augmentation considérable de la quantité de matière organique digérée dans l'intestin a probablement été rendue possible par un accroissement tissulaire de l'intestin grêle (Tulloh, 1966). Toutefois, la baisse de digestibilité dans le rumen n'a pas été totalement compensée au niveau intestinal, même pour l'amidon, ce qu'avaient observé Wheeler, Noller et Coppock (1975). Différents facteurs peuvent limiter sa digestion dans l'intestin grêle : une accélération du transit lorsque le niveau d'ingestion est élevé, une capacité de dégradation enzymatique insuffisante (Huber et al., 1961), peut-être en raison de conditions physico-chimiques ne permettant pas une activité maximale de l'amylase pancréatique (Wheeler et Noller, 1977), ou la limitation de l'amylolyse en raison d'une dégradation insuffisante des parois végétales. De plus, les activités amylolytiques et cellulolytique du gros intestin sont probablement réduites en raison d'un transit accéléré dû au niveau d'ingestion élevé (Grovum et Hecker, 1973). 


\section{Conclusion.}

Ces données relatives à l'influence du stade physiologique sur le comportement alimentaire et la digestion des aliments chez la vache laitière ont permis de mettre en évidence l'effet prépondérant de l'augmentation du niveau alimentaire mais aussi un effet propre lié aux conditions physiologiques et nutritionnelles de cette période. L'influence de facteurs physiques (volume des compartiments, vitesse de transit) ou liés aux besoins de l'animal (nutrition azotée) doit cependant être précisée dans des études ultérieures, afin de déterminer la part de variation due à ces différents facteurs.

Reçu en mars 1981.

Accepté en octobre 1981.

Remerciements. - Nous remercions vivement R. Pion et J. Prugnaud (Laboratoire d'Etude du Métabolisme azoté) qui ont permis la réalisation des dosages d'acides aminés.

\section{Références}

BATH I. H., ROOK J. A. F., 1963. Evaluation of cattle foods and diets in terms of the ruminal concentration of volatile fatty acids. J. agric. Sci., 61, 341-348.

BALDWIN R. L., KOONG L. J., ULYATT M. J., 1977. A dynamic model of ruminant digestion for evaluation of factors affecting nutritive value. Agric. Syst., 2, 255-288.

BAUMGARDT B. R., 1970. Control of feed intake in the regulation of energy balance, 235-253. In PHILLIPSON A. T. Physiology of digestion and metabolism in the ruminant. Ed. Oriel Press, Newcastle, U.K.

CAMPLING R. C., 1966. A preliminary study of the effect of pregnancy and of lactation on the voluntary intake of food by cows. Brit. J. Nutr., 20, 25-39.

CAMPLING R. C., FREER M., BALCH C. C., 1961. Factors affecting voluntary intake of food by cows. 2. The relationship between the voluntary intake of roughages, the amount of digesta in the reticulorumen and the rate of disappearance of digesta from the alimentary tract. Brit. J. Nutr., 19, 531-540.

COCIMANO M. R., LENG R. A., 1967. Metabolism of urea in sheep. Brit. J. Nutr., 21, 253-371.

CRAWFORD R. J., HOOVER W. H., JUNKINS L. L., 1980. Effects of solids and liquid flows on fermentation in continuous cultures. II. Nitrogen partition and efficiency of microbial synthesis. J. anim. Sci, 51, 986-995.

CRAWFORD R. J., HOOVER W. H., KNOWLTON P. H., 1980. Effects of solids and liquid flows on fermentation in continuous cultures. I. Dry matter and fiber digestion, VFA production and protozoa numbers. J. anim. Sci, 51, 975-985.

DULPHY J. P., 1971. Influence du poids vif et du niveau d'ingestion sur le comportement alimentaire et mérycique du mouton. Ann. Zootech., 20, 477-486.

DULPHY J. P., DEMARQUILLY C., HENRY M., 1975. Perte de composés volatils lors de la détermination à l'étuve de la teneur en matière sèche des ensilages. Ann. Zootech., 24, 743756.

EVERSON R. A., JORGENSEN N. A., CROWLEY J. W., JENSEN E. L., BARRINGTON G. P., 1976. Input-output of dairy cows fed a complete ration of a constant or variable forage-to-grain ratio. J. Dairy Sci., 59, 1776-1787.

FELL B. F., CAMPBELL R. M., MACKIE W. S., WEEKES T. E. C., 1972. Changes associated with 
pregnancy and lactation in some extra-reproductive organs of the ewe. J. agric. Sci., 79, 397 407.

FLATT W. P., COPPOCK C. E., MOORE L. A., 1965. Energy balance studies with lactating, non pregnant dairy cows consuming rations with varying hay to grain ratios, 121-130. In BLAXTER K. L., Energy metabolism. Acad. Press, London, New York.

FLATT W. P., MOE P. W., MOORE L. A., 1969. Influence of pregnency and ration composition on energy utilization by dairy cows, 123-136. In BLAXTER K. L., KIELANOWSKI J., THORBEK G., Energy metabolism of farm animals. Oriel Press, Newcastle, U.K.

FREER M., CAMPLING R. C., BALCH C. C., 1962. Factors affecting the voluntary intake of food by cows. 4 . Behaviour and reticular motility of cows receiving diets of hay, oat straw and oat straw with urea. Brit. J. Nutr., 16, 279-295.

GROVUM W. L., HECKER J. F., 1973. Rate of passage of digesta in sheep. 2. The effect of level of food intake on digesta retention times and on water and electrolyte absorption in the large intestine. Brit. J. Nutr., 30, 221-230.

HARRISON D. G., BEEVER D. E., THOMSON D. J., OSBOURN D. F., 1975. Manipulation of rumen fermentation in sheep by increasing the rate of flow of water from the rumen. $J$. agric., Sci., 85, 93-101.

HARTNELL G. F., SATTER L. D., 1978. Determination of rumen fill retention time and ruminal turnover rates of ingesta at different stages of lactation in dairy cows. J. anim. Sci., 48, 381392.

HUBER J. T., JACOBSON N. L., MAC GILLIARD A. D., ALLEN R. S., 1961. Utilization of carbohydrates introduced directly into the omaso-abomasal area of the stomach of cattle of various ages. J. Dairy Sci, 44, 321-330.

ISAACSON H. R., HINDS F. C., BRYANT M. P., OWENS F. N., 1975. Efficiency of energy utilization by mixed rumen bacteria in continuous culture. J. Dairy Sci., 58, 1645-1659.

JARRIGE R., JOURNET M., VERITE R., CHAMPREDON C., PION R., 1978. Azote, 89-128. In Alimentation des ruminants, Ed. INRA Publications, Versailles.

JOURNET M., REMOND B., 1976. Physiological factors affecting the voluntary intake of feed by cows : a review. Livest. Prod. Sci., 3, 129-146.

JOURNET M., VERITE R., 1979. Predicting equations of $\mathbf{N}$ duodenal flow in dairy cattle. Effects of level of feeding and proportion of concentrate in the diet. Ann. Rech. vet., 10, 303-306.

KANG-MEZNARICH J. H., BRODERICK G. A., 1980. Effects of incremental urea supplementation on ruminal ammonia concentration and bacterial protein formation. J. anim. Sci., 51, $422-431$.

LAMBERTH J. L., 1969. The effect of pregnancy in heifers on voluntary intake, total rumen contents, digestibility and rate of passage. Austr. J. exp. Agr. anim. Husb., 9, 493-496.

LAUGHREN L. C., YOUNG A. W., 1979. Duodenal nitrogen flow in response to increasing dietary crude protein in sheep. $J$. anim. Sci., 49, 211-220.

MATHIESON J., 1970. The automated estimation of chromic oxide. Proc. Nutr. Soc., 29, $30 \mathrm{~A}-31 \mathrm{~A}$.

MOORE S., SPACKMANN D. H., STEIN W. H., 1958. Chromatography of amino acids on sulfonated polystyrene resins. An improved method. Anal. Chem., 30, 1185-1190.

PION R., CHAMPREDON C., THIVEND P., 1973. Digestion des protéines de céréales chez l'agneau en croissance. Ann. Biol. anim. Bioch. Biophys., 13, 772-774.

REYNE Y., PRUD'HON M., DEBICKI A. M., GOUSSOPOULOS J., 1978. Caractéristiques des consommations d'aliments solide et liquide chez la lapine gestante puis allaitante nourrie ad libitum. Ann. Zootech., 27, 211-223.

RUCKEBUSCH Y., 1963. Recherches sur la régulation centrale du comportement alimentaire chez les ruminants. Th. Doc. Sci. nat., Univ. Lyon, 213 p.

SATTER L. D., SLYTER L. L., 1974. Effect of ammonia concentration on rumen microbial protein production in vitro. Brit. J. Nutr., 32, 199-208.

SIEBERT B. D., ALLDEN W. G., 1979. Nutrient flow and weight change in grazing cattle. Ann. Rech. vet., 10, 326-328.

SUTTON J. D., BROSTER W. H., SCHULLER E., SMITH T., NAPPER D. J., 1977. Long term effect of level of intake and diet composition on the performance of lactating dairy cows. 3. Milk composition and rumen fermentation. Proc. Nutr. Soc., 36, 147 A. 
SUZUKI S., FUJITA H., SHINDE Y., 1969. Change in the rate of eating during a meal and the effect of the interval between meals on the rate at which cows eat roughages. Anim. Prod., 11, 29-41.

TAMMINGA S., VAN DER KOELEN C. J., VAN VUUREN A. M., 1979. Effect of the level of feed intake on nitrogen entering the small intestine of dairy cows. Livest. Prod. Sci., 6, 255-262.

THIVEND P., MERCIER C., GUILBOT A., 1972. Determination of starch with glucoamylase, 79-82. In Methods in carbohydrate chemistry, Vol. VI, WHISTLER R. L., BEMILLER J. N. Acad. Press, London.

TISSERAND J. L., COLEOU J., ZELTER Z., 1962. Emploi du sesquioxyde de chrome comme indicateur dans les études de bilan digestif chez le ruminant. C. R. Acad. Sci. sér. D, Paris, 254, 2233-2235.

TULLOH N. M., 1966. Physical studies of the alimentary tract of grazing cattle. IV. Dimensions of the tract in lactating and non lactating cows. N. Z. J. Agric. Res., 9, 999-1008.

TYRRELL H. F., MOE P. W., 1972. Net energy value for lactation of a high and low concentrate ration containing corn silage. J. Dairy Sci., 55, 1106-1112.

TYRRELL H. F., MOE P. W., 1975. Effect of intake on digestive efficiency. J. Dairy Sci., 58, 1151-1163.

ULYATT M. J., BLAXTER K. L., MACDONALD I., 1967. The relations between the apparent digestibility of roughages in the rumen and lower gut of sheep, the volume of fluid in the rumen and voluntary feed intake. Anim. Prod., 9, 463-470.

VAN EENAEME C., BIENFAIT J. M., LAMBOT O., PONDANT A., 1969. Détermination automatique de l'ammoniaque dans le liquide du rumen par la méthode de Berthelot adaptée à l'auto-analyzer. Ann. Méd. vét., 7, 419-429.

VAN'T KLOOSTER A. T., KEMP A., GEURINK J. H., ROGERS P. A. M., 1972. Studies on the amount and composition of digesta flowing through the duodenum of dairy cows. I. Rate of flow of digesta measured direct and estimated indirect by the indicator dilution technique. Neth. J. agric. Sci, 20, 314-324.

WESTON R. H., 1979. Digestion during pregnancy and lactation in sheep. Ann. Rech. vét., 10. 442-444.

WHEELER W. E., NOLLER C. H., COPPOCK C. E., 1975. Effect of forage to concentrate ratio in complete feeds and feed intake on digestion of starch by dairy cows. J. Dairy Sci, 58, $1902-$ 1906.

WHEELER W. E., NOLLER C. H., 1977. Gastro-intestinal tract $\mathrm{pH}$ and starch in feces of ruminants. J. anim. Sci., 44, 131-135. 